\title{
Predicted formation of superconducting platinum-hydride crystals under pressure in the presence of molecular hydrogen
}

\author{
Duck Young Kim ${ }^{1}$, Ralph H. Scheicher ${ }^{2}$, Chris J. Pickard ${ }^{3}$, R. J. Needs 1 $* *$ and R. Ahuja ${ }^{2,4}+$ \\ 1 Theory of Condensed Matter Group, \\ Cavendish Laboratory, J J Thomson Avenue, \\ Cambridge CB3 OHE, United Kingdom \\ ${ }^{2}$ Condensed Matter Theory Group, Department of Physics and Astronomy, \\ Uppsala University, P.O. Box 516, SE-751 20, Uppsala, Sweden \\ ${ }^{3}$ Department of Physics and Astronomy, University College London, \\ Gower St, London WC1E 6BT, United Kingdom \\ ${ }^{4}$ Department of Materials and Engineering, Applied Materials Physics, \\ Royal Institute of Technology (KTH), SE-100 44 Stockholm, Sweden
}

\begin{abstract}
Noble metals adopt close-packed structures at ambient pressure and rarely undergo structural transformation at high pressures. Platinum $(\mathrm{Pt})$, in particular, is normally considered to be unreactive and is therefore not expected to form hydrides under pressure. We predict that platinum hydride (PtH) has a lower enthalpy than its constituents solid Pt and molecular hydrogen at pressures above $21.5 \mathrm{GPa}$. We have calculated structural phase transitions from tetragonal to hexagonal close-packed or face-centered cubic (fcc) PtH between 70 and $80 \mathrm{GPa}$. Linear response calculations indicate that $\mathrm{PtH}$ is a superconductor at these pressures with a critical temperature of about 10-25 K. These findings help to shed light on recent observations of pressure-induced metallization and superconductivity in hydrogen-rich materials. We show that formation of fcc metal hydrides under pressure is common among noble metal hydrides and examine the possibility of superconductivity in these materials.
\end{abstract}

PACS numbers: 63.20.kd, 74.25.Jb, 74.10.+v, 74.62.Fj

*Electronic address: rn11@cam.ac.uk

†Electronic address: rajeev.ahuja@fysik.uu.se 
Pt maintains the face-centered cubic crystal structure up to at least $304 \mathrm{GPa}$ [1], which covers most of the range currently accessible in static diamond anvil cell (DAC) experiments, and makes it suitable for use as a pressure standard [2, 3]. Pt is frequently used to form electrodes (which allow measurements of electrical resistance, the concentration of electrical carriers and the Hall mobility) and as a heat coupler. As a noble metal, Pt is fairly unreactive at ambient conditions, and this has generally been assumed also to hold at high pressures. However, chemical reactions of Pt with other materials present in the sample chamber can lead to the unexpected formation of new compounds as, for example, described by Gregoryanz et al. [4] who observed formation of Pt nitride when heating nitrogen with a piece of platinum.

The hydrogen-dense material silane $\left(\mathrm{SiH}_{4}\right)$ forms molecular crystals at low pressures. Two experimental studies of the metallization of $\mathrm{SiH}_{4}$ under pressure have recently appeared [5, 6]. The first study [6] provided optical evidence for metallization of $\mathrm{SiH}_{4}$ above $60 \mathrm{GPa}$. Remarkably, the second study by Eremets et al. [5] reported superconductivity of $\mathrm{SiH}_{4}$ in the pressure range 60-150 GPa with a superconducting transition temperature as high as $17 \mathrm{~K}$. These experimental findings have motivated a number of theoretical investigations of compressed $\mathrm{SiH}_{4}$ [7-9] suggesting that metallization and/or superconductivity can be achieved under pressure, but a clear explanation of the observations has not yet been achieved. While the above two experimental studies [5, 6] reported metallization of $\mathrm{SiH}_{4}$ above $60 \mathrm{GPa}$, whereas some other experiments claimed decomposition, amorphization, and the formation other metal-hydrogen alloys besides silane [10, 11]. This complexity itself is associated with hydrogen as being extremely light and mobile, as well as extraordinarily reactive. The properties of hydrogen are becoming drastically enhanced under high pressure and temperature, and make the interpretation of experimental data for hydrides [12-14] rather inconclusive.

Degtyareva et al. [11] have speculated that the formation of $\mathrm{PtH}$ under high pressures might explain some of the observations reported in Ref. 5, and they provided indirect evidence to support the hypothesis of PtH formation. Moreover, it appears likely that the silane in the experiments of Eremets et al. [5] partially decomposed, leading to release of hydrogen. Indeed $\mathrm{SiH}_{4}$ is a metastable compound at low pressures which is thermodynamically unstable to decomposition into $\mathrm{H}_{2}$ and $\mathrm{Si}$ [11], so that release of hydrogen might occur when the compression is sufficient to break bonds in the $\mathrm{SiH}_{4}$ molecules. Released hydrogen may then have reacted with the $\mathrm{Pt}$ electrodes to form $\mathrm{PtH}$ as the pressure was further increased.

Confirming the formation of superconducting $\mathrm{PtH}$ at high pressures would change the inter- 
pretation of the data of Eremets et al. [5], but would in no way diminish the importance of the experiments. In the new interpretation these experiments will have led to the discoveries of superconducting $\mathrm{PtH}$ under pressure and a new insulating phase of $\mathrm{SiH}_{4}$, and to the understanding that compressed $\mathrm{SiH}_{4}$ is prone to decomposition. Indeed, Degtyareva et al. [11] have suggested that hydrogen release from $\mathrm{SiH}_{4}$ at high pressures could be useful in synthesising metal hydrides.

The prevalence of DAC measurements and the steadily growing interest in high-pressure experimental studies of metal hydrides raises the possibility of decomposition and hydrogen release in such experiments. Inadvertent and undetected formation of noble metal hydrides could lead to erroneous pressure estimates and other effects which could substantially alter the interpretation of the data. A careful investigation of the possibility of pressure-induced reactions between hydrogen and noble metals such as $\mathrm{Pt}$ is therefore very important and timely.

We have used $a b$ initio random structure searching (AIRSS) to predict crystal structures of $\mathrm{PtH}$ under pressure [15]. This approach has been used to predict the structures of materials such as hydrogen [16], metal hydrides [17], transition metals [18], and molecular crystals [19-21]. In this study we have used the simplest version of the AIRSS technique in which an ensemble of structures is prepared by generating unit cells of random shapes with reasonable volumes into which the atoms are placed at random positions. The cell shapes and atomic positions are then relaxed to an enthalpy minimum at fixed pressure. We studied simulation cells containing 1, 2, 4, 6, and 8 formula units of $\mathrm{PtH}$. We found three particularly low-enthalpy metallic structures of PtH (the corresponding space groups are given in parentheses): fcc $(F m \overline{3} m)$, hcp $\left(P 6_{3} / m m c\right)$, and tetragonal $(I \overline{4} m 2)$.

We have assumed that $\mathrm{PtH}$ forms in the 1:1 stoichiometry. The fcc structure was used for Pt and an hcp molecular form for hydrogen [16], in which the $\mathrm{H}_{2}$ molecules are randomly oriented in space. The variation of the enthalpies $H$ of the fcc, hcp, and tetragonal phases of PtH relative to that of $\mathrm{Pt}+\frac{1}{2} \mathrm{H}_{2}$ is given in Fig. 1, which shows that $\mathrm{PtH}$ is more stable at pressures above approximately $20 \mathrm{GPa}$. We also calculated the dynamical stability of the PtH structures and evaluated their electron-phonon coupling (EPC) strengths, $\lambda$, in order to study the possibility of phonon-mediated superconductivity in PtH. Since the zero-point motion of the hydrogen nuclei is substantial, we have included effects from the zero-point enthalpy (ZPE) calculated within the quasiharmonic approximation. For details of the computational methods we refer the reader to the supplementary material [22]. It should be noted that there are alternative ways of calculating the superconducting transition temperature $T_{c}$. In particular, there has recently been considerable progress within the 
framework of density functional theory in developing truly ab initio quantitative predictions of $T_{c}$ in the absence of adjustable parameters, which combine electron-phonon coupling and Coulomb interactions [23-28].

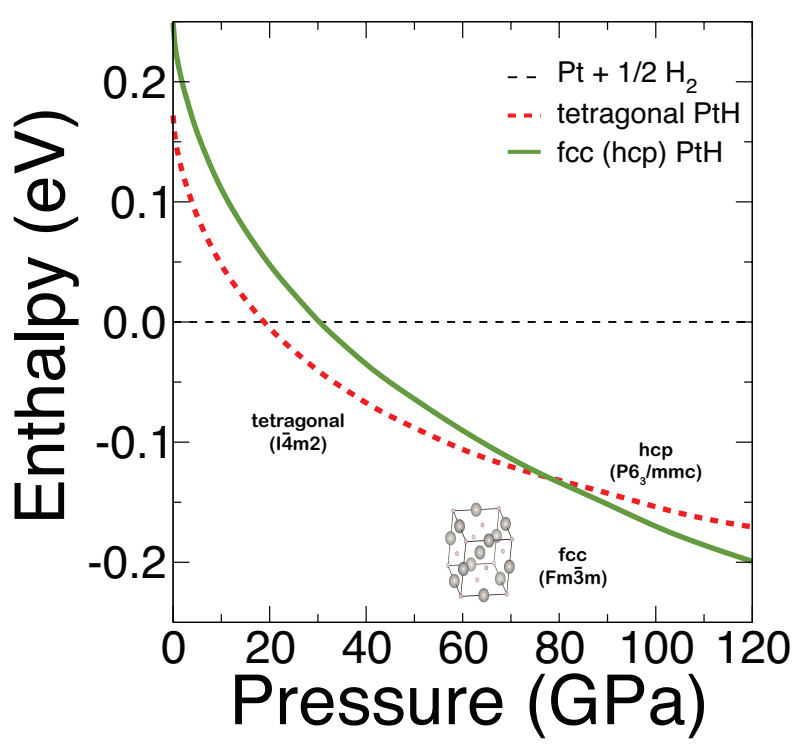

FIG. 1: (color online). Comparison of the enthalpies of $\mathrm{Pt}+\frac{1}{2} \mathrm{H}_{2}$ and the tetragonal and fcc phases of PtH.

Figure 1 shows that it is thermodynamically favourable for tetragonal $\mathrm{PtH}$ to form from elemental $\mathrm{Pt}$ and $\mathrm{H}_{2}$ above $18.7 \mathrm{GPa}$ when the zero-point energy of the nuclei is neglected (above 21.5 GPa when the zero-point energy contribution is included; see the supplementary material [22]). Experimentally, a tetragonal $\mathrm{PtH}$ was observed at room temperature, $26 \mathrm{GPa}$ on compression and $20 \mathrm{GPa}$ on decompression [29]. We find a transition from tetragonal to hcp or fcc PtH between 70 and $80 \mathrm{GPa}$ (Fig. 1). The fcc and hcp structures differ only in the stacking of layers, making them virtually degenerate in energy (to within a few meV per formula unit). Under pressure, a previous calculation using a simple model suggested that the site occupied by hydrogen in $d$-metal monohydrides tends to change from the tetrahedral to the octahedral site and that the hydride transforms into the hcp or fcc structure [30], in good agreement with our findings. In the present context it is more likely for fcc $\mathrm{PtH}$ to form, since the $\mathrm{Pt}$ atoms as the heavier host material are arranged in that structure prior to exposure to hydrogen. Experimentally hcp $\mathrm{PtH}$ not fcc $\mathrm{PtH}$ was observed and we therefore speculate that kinetics might favor a sequence of structural transformation from tetragonal $\mathrm{PtH}$ to hcp $\mathrm{PtH}$.

Having established the likely formation of $\mathrm{PtH}$ from $\mathrm{Pt}$ and $\mathrm{H}_{2}$ in the pressure range relevant for the experimental observations reported in Ref. 5, we evaluated the electron-phonon coupling 
(EPC) strength in PtH to examine its potential for superconductivity, in a similar fashion to our studies of metal tri-hydrides [31, 34], as summarized in the supplementary material [22]. This study is crucial because the formation of superconducting $\mathrm{SiH}_{4}$ was a central claim of Ref. 5, and perhaps superconducting PtH could provide an alternative explanation.

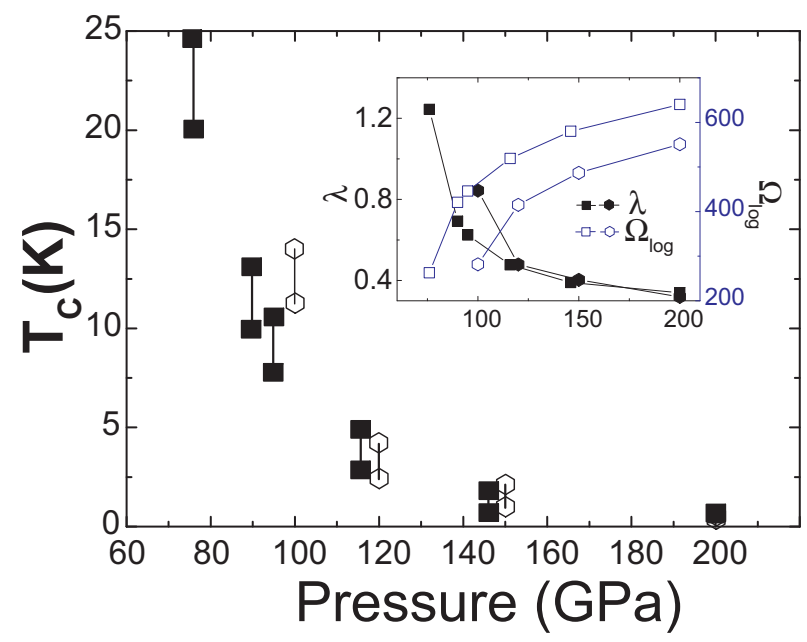

FIG. 2: (color online). Superconducting transition temperature $T_{c}$ of $\mathrm{PtH}$ as a function of pressure. Filled squares and hollow hexagons show values of $T_{c}$ for the fcc and hep structures, respectively, with the higher $T_{c}$ value at each pressure corresponding to the choice $\mu^{*}=0.10$ and the lower $T_{c}$ value to the choice $\mu^{*}=0.13$. The inset displays the evolution with pressure of the electron-phonon coupling parameter, $\lambda$, and the asymptotic phonon momentum $\Omega_{l o g}$ (see the supplementary material [22]). The fcc phase is dynamically unstable below $77 \mathrm{GPa}$ and the hcp phase is dynamically unstable below $100 \mathrm{GPa}$.

Papaconstantopoulos suggested that PtH could be superconducting under ambient conditions [35], but subsequent experiments found no evidence of PtH formation at low pressure [29, 41]. Tetragonal $\mathrm{PtH}$ becomes more stable than $\mathrm{Pt}+\frac{1}{2} \mathrm{H}_{2}$ above $20 \mathrm{GPa}$. We calculated the EPC of tetragonal $\mathrm{PtH}$ in the pressure range 20-60 GPa and found no significant coupling, so it is not likely that superconducting tetragonal PtH could exist. Figure 2 shows the variation of the calculated superconducting transition temperature $T_{c}$ of $\mathrm{PtH}$ with pressure. In the whole pressure interval of 80-200 GPa we used a fixed value for the effective Coulomb interaction parameter $\mu^{*}=0.13$, as has been common procedure [13, 36]. However, we also explored the effects on $T_{c}$ of changing $\mu^{*}$ to 0.10 [12, 13]. With this, we have covered the two extreme choices of $\mu^{*}$, resulting in corresponding high- and low-estimates of $T_{c}$ (Fig. 2). It is worth noting that the value of $\mu^{*}$ may also be chosen to vary as a function of the electronic density of states at the Fermi level [39]. 
The highest value of $T_{c}$ is found in the vicinity of the onset of dynamical stability of the structures, as expected for phonon-mediated superconductivity [31-33]. The fcc structure is dynamically stable above $77 \mathrm{GPa}$ where it possesses the highest $T_{c}$ of around $25 \mathrm{~K} . T_{c}$ decreases monotonically and quite rapidly as the pressure rises, as shown in Fig. 2] and reaches essentially zero around 140 GPa. The hcp structure is dynamically stable above $100 \mathrm{GPa}$ (and is almost degenerate in enthalpy with fcc $\mathrm{PtH}$ ) and its maximum $T_{c}$ of around $15 \mathrm{~K}$ occurs at $100 \mathrm{GPa}$.
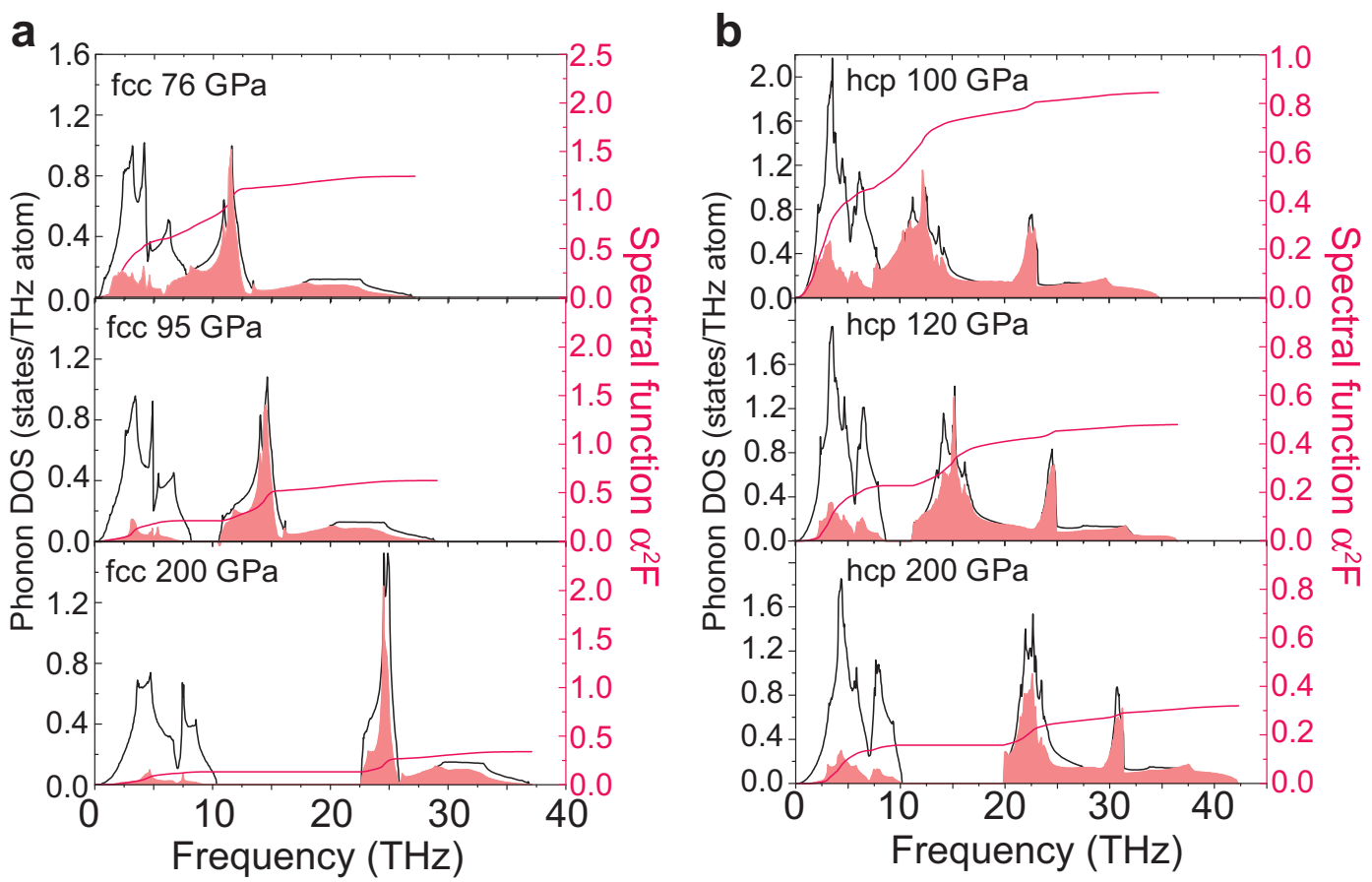

FIG. 3: (color online). Spectral function $\alpha^{2} F$ (red shaded areas), the integral of the spectral function up to frequency $\omega\left(\int_{0}^{\omega} \alpha^{2} F\left(\omega^{\prime}\right) / \omega^{\prime} d \omega^{\prime}\right)$ (red line), and the phonon density of states (solid black line) as a function of frequency at selected pressures for (a) fcc PtH and (b) hcp PtH.

Figure 3 shows the spectral function $\alpha^{2} F$ and the phonon density of states (PhDOS) at different pressures for both fcc and hcp PtH. At the lowest pressure at which the fcc phase is stable (77 GPa), the Pt and $\mathrm{H}$ atoms both contribute to the EPC (Fig. 3a). With increasing pressure, the spectral function splits into high and low frequency bands as shown in the second and third panels of Fig. 3a, and only hydrogen vibrations contribute to the EPC in the higher pressure band. The situation is similar in the hcp phase (Fig. 3b), in the sense that the respective contributions to the EPC parameter $\lambda$ from $\mathrm{Pt}$ and $\mathrm{H}$ are nearly the same as at lower pressures. With increasing pressure, the integrated $\lambda$ decreases as the phonon frequencies increase. This behavior can be understood from Eq. 3 of the supplementary material [22], where it can be seen that the spectral function $\alpha^{2} F$ 
is divided by the phonon frequencies.

Interestingly, Eremets et al. reported an apparent divergence in $T_{c}$ between 80 and $100 \mathrm{GPa}$ [5] which coincides with our calculated transition pressure between fcc and hcp PtH. These structural transformations are accompanied by softening of the acoustic branch modes which is a precursor of structural transformation. High-pressure experiments on PtH have been reported earlier [29], but since they were performed at room temperature, no information regarding possible superconductivity could be obtained. It would be very interesting to study compressed $\mathrm{PtH}$ at temperatures below $25 \mathrm{~K}$ to determine whether it is indeed superconducting.

At low pressures, fcc-Pt absorbs hydrogen and forms tetragonal $\mathrm{PtH}$, which is a distortion of the fcc structure. Under pressure, the tetragonal $\mathrm{PtH}$ phase is restored to its original fcc structure or the similar close-packed hcp structure, which possess strong EPC and hence a high superconducting critical temperature. Such a structural transformation from a lower-symmetry phase to the fcc structure also applies to other hydrides formed by metals which are neighbors of $\mathrm{Pt}$ in the periodic table (referred to in the following as the Pt family of hydrides). We investigated the metals Rh, $\mathrm{Pd}, \mathrm{Ag}, \mathrm{Ir}$, and $\mathrm{Au}$, which all adopt the fcc structure at ambient pressure and have extremely small or zero superconducting $T_{c}$, and determined the onset pressure at which the corresponding fcc hydrides become dynamically stable. We find that fcc-RhH (lattice constant $a=4.02 \AA$ ) and fcc$\mathrm{PdH}(a=4.08 \AA)$ are stable at almost ambient pressure, consistent with experimental observations $[40,42,43,45]$. Also, our calculations are in good agreement with the measured lattice constants which were reported to be $4.01 \AA$ at $3.8 \mathrm{GPa}$ (hydrogen gas pressure) for fcc-RhH [42, 43] and $4.09 \AA$ at ambient pressure for $\mathrm{PdH}$ [45]. The metal hydrides fcc-AgH $(a=4.00 \AA)$ and fcc-IrH $(a$ $=3.88 \AA$ ) are dynamically stable at 50 and $80 \mathrm{GPa}$, respectively. Dynamical stability of fcc-AuH ( $a=3.80 \AA$ ) is achieved only above $220 \mathrm{GPa}$. We emphasize that other hydride structures besides fcc might be stable at lower pressures.

Similarly to PtH, the dynamical stability of other fcc-structure hydrides can be regarded as precursors for superconductivity, while lower symmetric structures might form at much lower pressures [44]. Figure 4 shows the calculated $T_{c}$ at the onset of dynamical stability of the fcc structure in the Pt family of hydrides. $\mathrm{PdH}$ is calculated to possess a $T_{c}$ of $20 \mathrm{~K}$, which is compatible with experiments on non-stoichiometric $\mathrm{PdH}_{x}$ which reported a $T_{c}$ of $9 \mathrm{~K}$ [45], although we note that our results do not reproduce the observed isotope dependence of $T_{c}$ in $\mathrm{PdH} / \mathrm{PdD}[14]$. We predict $\mathrm{IrH}$ to have a $T_{c}$ of $7 \mathrm{~K}$ at $80 \mathrm{GPa}$. Remarkably, the $T_{c}$ of fcc-AuH is estimated to be $21 \mathrm{~K}$, however, it takes a very high pressure of $220 \mathrm{GPa}$ to reach this superconducting state. As 


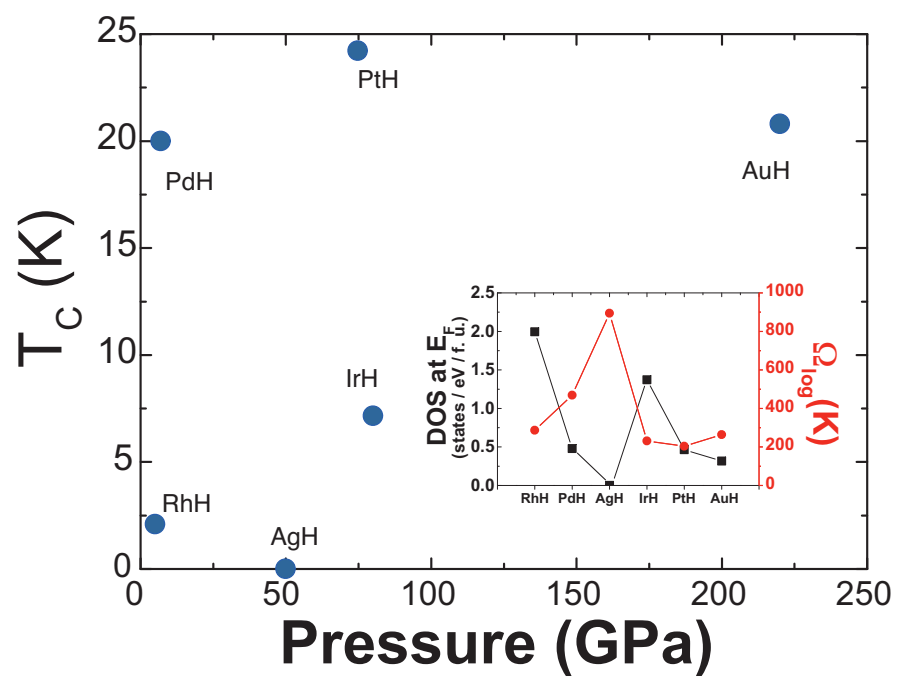

FIG. 4: (color online). Maximum $T_{c}$ (calculated at the onset of dynamical stability of the fcc phase) for the metal hydrides $\mathrm{RhH}, \mathrm{PdH}, \mathrm{AgH}, \mathrm{IrH}, \mathrm{PtH}$, and $\mathrm{AuH}$. Note that the corresponding elemental metals in their fcc form all possess either extremely small $T_{c}(\mathrm{Rh}$ and $\mathrm{Ir})$ or are not superconducting at all $(\mathrm{Pd}, \mathrm{Ag}, \mathrm{Pt}$, and $\mathrm{Au})$.

the pressure is increased, the $T_{c}$ of all these fcc-hydrides will decrease, analogously to the case of $\mathrm{PtH}$. We predict that $\mathrm{AgH}$ is not a superconductor, because the electronic density of states exhibits a small bandgap at the Fermi level. AgH does however possesses the highest $\Omega_{l o g}$, and therefore, if electrons were to be donated in a complex alloy, this could lead to a good superconducting material, such as $(\mathrm{PdAg}) \mathrm{H}_{x}$, possessing a $T_{c}$ of about $16 \mathrm{~K}$ [47]. $\mathrm{RhH}$ has a small $T_{c}$ and it is worth note that no superconductivity of $\mathrm{RhH}$ has been detected at $T_{c}>0.3 \mathrm{~K}$ [48].

In conclusion, we have shown from first principles that formation of $\mathrm{PtH}$ from $\mathrm{Pt}$ and $\mathrm{H}_{2}$ can occur under pressure. Based on the empirical Allen-Dynes equation (see supplementary material), the resulting product is predicted to be a superconductor. Throughout the studied pressure interval of 80-200 GPa, we used the effective Coulomb interaction parameter $\mu^{*}=0.13$, a value which has often been used [36]. We did, however, consider the effect of changing $\mu^{*}$ to 0.10 on the predicted $T_{c}$. If our prediction concerning the formation of superconducting $\mathrm{PtH}$ at high pressures would be experimentally confirmed then this might lead to new interpretation of the data of Eremets et al. [5]. That work would mark the discovery of superconducting PtH under pressure and a new insulating phase of $\mathrm{SiH}_{4}$, and to the understanding that compressed $\mathrm{SiH}_{4}$ is prone to decomposition. We expect these findings to be relevant to recent observations of pressure-induced metallization 
and superconductivity in hydrogen-rich materials. Indeed, our calculations show that the formation of superconducting fcc metal hydrides under pressure may be a common phenomenon among noble metal hydrides. The pressure-induced formation of $\mathrm{PtH}$ and of other noble metal hydrides, along with their associated superconductivity, represents an important aspect which should be considered in the interpretation of diamond anvil cell experiments whenever the corresponding metals and hydrogen are present, with the latter introduced either directly or entering via decomposition of hydrides.

The authors acknowledge financial support from Wenner-Gren Foundations, the Swedish Research Council (VR, Grant No. 621-2009-3628), Formas, and the Engineering and Physical Sciences Research Council of the UK. The Swedish National Infrastructure for Computing (SNIC) and the Uppsala Multidisciplinary Center for Advanced Computational Science (UPPMAX) provided computing time.

[1] R. Ahuja, P. Söderlind, J. M. Wills, B. Johansson, and O. Eriksson, High Pressure Research 12, 161 (1994).

[2] E. Y. Tonkov and E. G. Ponyatovsky, Phase Transformations of Elements Under High Pressure (CRC Press, Boca Raton, 2005), pp. 273-275.

[3] N. Holmes, J. Appl. Phys. 66, 2962 (1989).

[4] E. Gregoryanz, C. Sanloup, M. Somayazulu, J. Badro, G. Fiquet, H.-k. Mao, and R. J. Hemley, Nature Mater. 3, 294 (2004).

[5] M. I. Eremets, I. A. Trojan, S. A. Medvedev, J. S. Tse, and Y. Yao, Science 319, 1506 (2008).

[6] X. J. Chen, V. V. Struzhkin, Y. Song, A. F. Goncharov, M. Ahart, Z. X. Liu, H.-k. Mao, and R. J. Hemley, Proc. Natl. Acad. Sci. U.S.A. 105, 20 (2008).

[7] D. Y. Kim, R. H. Scheicher, S. Lebègue, J. Prasongkit, B. Arnaud, M. Alouani, and R. Ahuja, Proc. Natl. Acad. Sci. U.S.A. 105, 16454 (2008).

[8] X.-J. Chen, J.-L. Wang, V. V. Struzhkin, H.-k. Mao, R. J. Hemley, and H.-Q. Lin, Phys. Rev. Lett. 101, 077002 (2008).

[9] M. Martinez-Canales, A. R. Oganov, Y. Ma, Y. Yan, A. O. Lyakhov, and A. Bergara, Phys. Rev. Lett. 102, 087005 (2009).

[10] C. Narayana, R. H. Greene, and A. L. Ruoff, J. Phys: Conf. Ser. 121, 042019 (2008). 
[11] O. Degtyareva, J. E. Proctor, C. L. Guillaume, E. Gregoryanz, and M. Hanfland, Solid State Communications 149, 1583 (2009).

[12] B. Baranowski, Top. Appl. Phys. 29, 157 (1978).

[13] B. Baranowski, S. Majchrzak, and T.B. Flanagan, J. Phys. F 1, 258 (1971).

[14] E. G. Ponyatovskii, V. E. Antonov, and I. T. Belash, in "Problems in Solids State Physics“, (A. M. Prokhorov and A. S. Prokhorov, Eds.), 109 MIR, Moscow (1984).

[15] C. J. Pickard and R. J. Needs, Phys. Rev. Lett. 97, 045504 (2006).

[16] C. J. Pickard and R. J. Needs, Nature Phys. 3, 473 (2007).

[17] C. J. Pickard and R. J. Needs, Phys. Rev. B 76, 144114 (2007).

[18] C. J. Pickard and R. J. Needs, J. Phys.: Condens. Matter 21, 452205 (2009).

[19] C. J. Pickard and R. J. Needs, Nature Mater. 7, 775 (2008).

[20] A. D. Fortes, E. Suard, M.-H. Lemee-Cailleau, C. J. Pickard, and R. J. Needs, J. Am. Chem. Soc. 131, 13508 (2009).

[21] P. Srepusharawoot, A. Blomqvist, C. M. Araújo, R. H. Scheicher, and R. Ahuja, Phys. Rev. B 82, 125439 (2010).

[22] See supplementary material at/http://link.aps.org/supplemental/10.1103/PhysRevLett.xxx.xx for details of the computational methods, crystal structures, and phonon enthalpy contributions.

[23] M. Lüders, M. A. L. Marques, N. N. Lathiotakis, A. Floris, G. Profeta, L. Fast, A. Continenza, S. Massidda, and E. K. U. Gross, Phys. Rev. B 72, 024545 (2005).

[24] M. A. L. Marques, M. Lüders, N. N. Lathiotakis, G. Profeta, A. Floris, L. Fast, A. Continenza, E. K. U. Gross, and S. Massidda, Phys. Rev. B 72, 024546 (2005).

[25] A. Floris, G. Profeta, N. N. Lathiotakis, M. Lüders, M. A. L. Marques, C. Franchini, E. K. U. Gross, A. Continenza, and S. Massidda, Phys. Rev. Lett. 94, 037004 (2005).

[26] G. Profeta, C. Franchini, N. N. Lathiotakis, A. Floris, A. Sanna, M. A. L. Marques, M. Lüders, S. Massidda, E. K. U. Gross, and A. Continenza, Phys. Rev. Lett. 96, 047003 (2006).

[27] A. Sanna, G. Profeta, A. Floris, A. Marini, E. K. U. Gross, and S. Massidda, Phys. Rev. B 75, 020511 (2007).

[28] A. Floris, A. Sanna, S. Massidda, and E. K. U. Gross, Phys. Rev. B 75, 054508 (2007).

[29] N. Hirao, F. Hiroshi, O. Yasuo, T. Kenichi, and K. Takumi, Acta Cryst. 64, C609 (2008).

[30] V. A. Somenkov, V. P. Glazkov, A. V. Irodov, and S. Sh. Shilstein, J. Less-Common Metals 129, 171 (1987). 
[31] D. Y. Kim, R. H. Scheicher, H.-k. Mao, T. W. Kang, and R. Ahuja, Proc. Natl. Acad. Sci. U.S.A. 107, 2793 (2010).

[32] Y. Yao, J. S. Tse, K. Tanaka, F. Marsiglio, and Y. Ma, Phys. Rev. B 79, 054524 (2009).

[33] D. Erskine, P. Y. Yu, K. J. Chang, and M. L. Cohen, Phys. Rev. Lett. 57, 2741 (1986).

[34] D. Y. Kim, R. H. Scheicher, and R. Ahuja, Phys. Rev. Lett. 103, 077002 (2009).

[35] D. A. Papaconstantopoulos, J. Less-Common Metals 73, 305 (1980).

[36] N. W. Ashcroft, Phys. Rev. Lett. 92, 187002 (2004).

[37] W. L. McMillan, Phys. Rev. 167, 331 (1968).

[38] P. B. Allen and R. C. Dynes, Phys. Rev. B 12, 905 (1975).

[39] K. H. Bennemann and J. W. Garland, AIP Conference Proceedings 4, 103 (1972).

[40] Y. Fukai, Metal-hydrogen system (Springer, Berlin and Heidelberg, 2003).

[41] A. Traverse, H. Bernas, J. Chaumont, L. Dumoulin, and M. Gupta, Solid State Commun. 40, 725 (1981).

[42] M. Tkacz, J. Chem. Phys. 108, 2084 (1998).

[43] V. E. Antonov, J. Alloys Compd. 330-332, 110 (2002).

[44] V. E. Antonov, T. E. Antonova, I. T. Belash, A. E. Gorodezkii, and E. G. Ponyatovskii, Dokl. Acad. Nauk. SSSR 266, 376 (1982).

[45] H. Hemmes, A. Driessen, R. Griessen, and M. Gupta, Phys. Rev. B 39, 4110 (1989).

[46] B. Stritzker and W. Buckel, Z. Physik 257, 1 (1972).

[47] W. Buckel and B. Stritzker, Phys. Lett. A 43, 403 (1973).

[48] V. E. Antonov, I. T. Belash, O. V. Zharikov, and A. V. Palnichenko, Phys. Stat. Sol. 142, K155 (1987). 


\section{Supplementary Material}

"Formation and superconductivity of platinum hydride under pressure"

D. Y. Kim, R. H. Scheicher, C. J. Pickard, R. J. Needs, and R. Ahuja

\section{A. Methods}

Our results were obtained using density-functional-theory (DFT) methods [1, 2] and the generalized gradient approximation (GGA) of Perdew, Burke and Ernzerhof for the exchangecorrelation functional [3, 4]. The structure searching was carried out with the CASTEP planewave code [5] and ultrasoft pseudopotentials [6]. For the searches we used a plane-wave basis-set cutoff of $550 \mathrm{eV}$ and a Brillouin-zone integration grid of spacing $2 \pi \times 0.07 \AA^{-1}$. The structures of interest were further relaxed for enthalpy comparisons, which were done with the VASP code [7, 8] with the projector-augmented wave (PAW) approach [9]. A cutoff energy of $700 \mathrm{eV}$ was found to produce converged results. The Monkhorst-Pack scheme [10] was used to generate the $k$-point grid meshes of $31 \times 31 \times 31$ for Pt and $\mathrm{PtH}(\Gamma$-centered in the case of hcp-PtH). For calculations of solid hydrogen, a large supercell containing $36 \mathrm{H}_{2}$ molecules was used; therefore, the Brillouinzone was only sampled at the $\Gamma$-point. Calculations of the phonon modes and frequencies were performed with density-functional perturbation theory using the QUANTUM ESPRESSO code [11]. The electronic orbitals were expanded in a plane-wave basis set with a kinetic energy cutoff of $60 \mathrm{Ry}$. The Brillouin zone (BZ) integrations in the electronic and phonon calculations were performed using Monkhorst-Pack meshes. We refer to meshes of $k$-points for electronic states and meshes of $q$-points for phonons. The EPC matrix elements were computed in the first BZ on a $8 \times 8 \times 8 q$-mesh $(8 \times 8 \times 4 q$-mesh $)$ using individual EPC matrices obtained with a $24 \times 24 \times 24$ $(24 \times 24 \times 16) k$-points mesh for fcc $($ hcp $)$. Convergence tests of the EPC calculations were performed with up to $12 \times 12 \times 12 q$-meshes for fcc and $8 \times 8 \times 8 q$-meshes for hcp.

The total phonon zero-point energy was calculated as $E_{p h}=\frac{1}{2} \sum_{i} \hbar \omega_{i}$ over a range of volumes $V$, where $\hbar \omega_{i}$ are the individual phonon energies. We fitted the results to a polynomial and differentiated with respect to $V$ to obtain the zero-point enthalpy as $H_{p h}=E_{p h}-V d E_{p h} / d V$.

$T_{c}$ was calculated using the Allen-Dynes equation,[12]

$$
T_{c}=\frac{\Omega_{l o g}}{1.2} \exp \left(-\frac{1.04(1+\lambda)}{\lambda-\mu^{*}(1+0.62 \lambda)}\right),
$$


with the standard value $\mu^{*}=0.13$. The normalized weighting function of the Eliashberg theory [13] is

$$
g(\omega)=\frac{2}{\lambda \omega} \alpha^{2} F(\omega)
$$

The parameter $\lambda$ is a dimensionless measure of the strength of $\alpha^{2} F$ :

$$
\lambda=2 \int_{0}^{\infty} d \omega \alpha^{2} F(\omega) / \omega
$$

and the logarithmic average frequency, $\Omega_{l o g}$ in units of $\mathrm{K}$, is

$$
\Omega_{l o g}=\exp \left(\int_{0}^{\infty} g(\omega) \ln \omega d \omega\right) .
$$


B. Crystal structure details

tetragonal PtH at 46.6 GPa

lattice vectors:

$a=b=3.422 \AA, c=2.782 \AA$

atomic positions:

Pt $1 / 2 \quad 0 \quad 3 / 4$

Pt $0 \quad 1 / 2 \quad 1 / 4$

H

H $1 / 21 / 2 \quad 1 / 2$

\section{fec PtH at 40.6 GPa}

lattice vector:

$a=2.843 \AA$

atomic positions:

Pt $1 / 2$ 1/2 1/2

$\mathrm{H} \quad 0 \quad 0 \quad 0$

\section{hcp PtH at 48.1 GPa}

lattice vectors:

$a=2.789 \AA, c=4.733 \AA$

atomic positions:

Pt 1/3 2/3 1/4

Pt 2/3 1/3 3/4

H $\quad 0 \quad 0 \quad 0 \quad 0$

$\begin{array}{llll}\mathrm{H} & 0 & 0 & 1 / 2\end{array}$ 


\section{Phonon enthalpy contribution}

Figure 5 shows the effect of the nuclear zero-point enthalpy (ZPE) $H_{p h}$, as defined in the Methods section, on the formation pressure of $\mathrm{PtH}$. The difference in total enthalpy between tetragonal $\mathrm{PtH}$ and $\mathrm{Pt}+\frac{1}{2} \mathrm{H}_{2}$ is plotted. As can be seen, the inclusion of $H_{p h}$ shifts the formation pressure up to $51.8 \mathrm{GPa}$, far beyond the $18.7 \mathrm{GPa}$ obtained when the ZPE is neglected. Such a shift seems unreasonably high, and we therefore decided to calculate the effect of $H_{p h}$ for a material with a well-established formation pressure.

$\mathrm{PdH}$ is known to form close to ambient pressure [14]. Figure 6]shows that the enthalpy comparison of $\mathrm{PdH}$ with $\mathrm{Pd}+\frac{1}{2} \mathrm{H}_{2}$ yields a formation pressure in the vicinity of $0 \mathrm{GPa}$, actually slightly below it. However, once $H_{p h}$ is included, the formation pressure shifts to $23 \mathrm{GPa}$ (Fig. 6), in contradiction with experiment.

What is the source of this discrepancy? The zero-point enthalpy $H_{p h}$ contributions from the metal and metal hydride can be rather precisely determined computationally, in particular because there is very little uncertainty about the slope $d E_{p h} / d V$, the phonon pressure term, which is multiplied by the volume and added to the phonon energy to yield the phonon enthalpy $H_{p h}$. For Pt and $\mathrm{PtH}$, the phonon energy $E_{p h}$ is found to depend linearly on the cell volume, and so $d E_{p h} / d V$ remains virtually constant throughout the pressure range studied. The main uncertainty stems from the contribution to $H_{p h}$ from $\mathrm{H}_{2}$, a system which is notoriously difficult to describe properly within density functional theory at low pressures [15]. As a consequence, it is hard to determine the phonon energy $E_{p h}$ accurately at low pressures, and furthermore, the non-linear dependence of $E_{p h}$ on $V$ in solid hydrogen (see Figure 7) prevents us from obtaining a reliable value of $d E_{p h} / d V$ at lower pressure.

To correct for the error in $E_{p h}$ and in the slope $d E_{p h} / d V$, we therefore determined a correction factor which multiplies $H_{p h}$ for $\mathrm{H}_{2}$, in order to recover the known formation pressure of $\mathrm{PdH}$. Figure 6 shows the enthalpy when $H_{p h}$ for $\mathrm{H}_{2}$ is scaled by a factor of 3.3, which yields a reasonable formation pressure in the vicinity of $0 \mathrm{GPa}$.

Applying the same scaling correction to $H_{p h}$ for $\mathrm{H}_{2}$ in the enthalpy comparison for $\mathrm{PtH}$ (Fig. 8), we obtain a formation pressure of $21.5 \mathrm{GPa}$, corresponding to a much more reasonable shift of 2.8 GPa due to the effects of ZPE. 


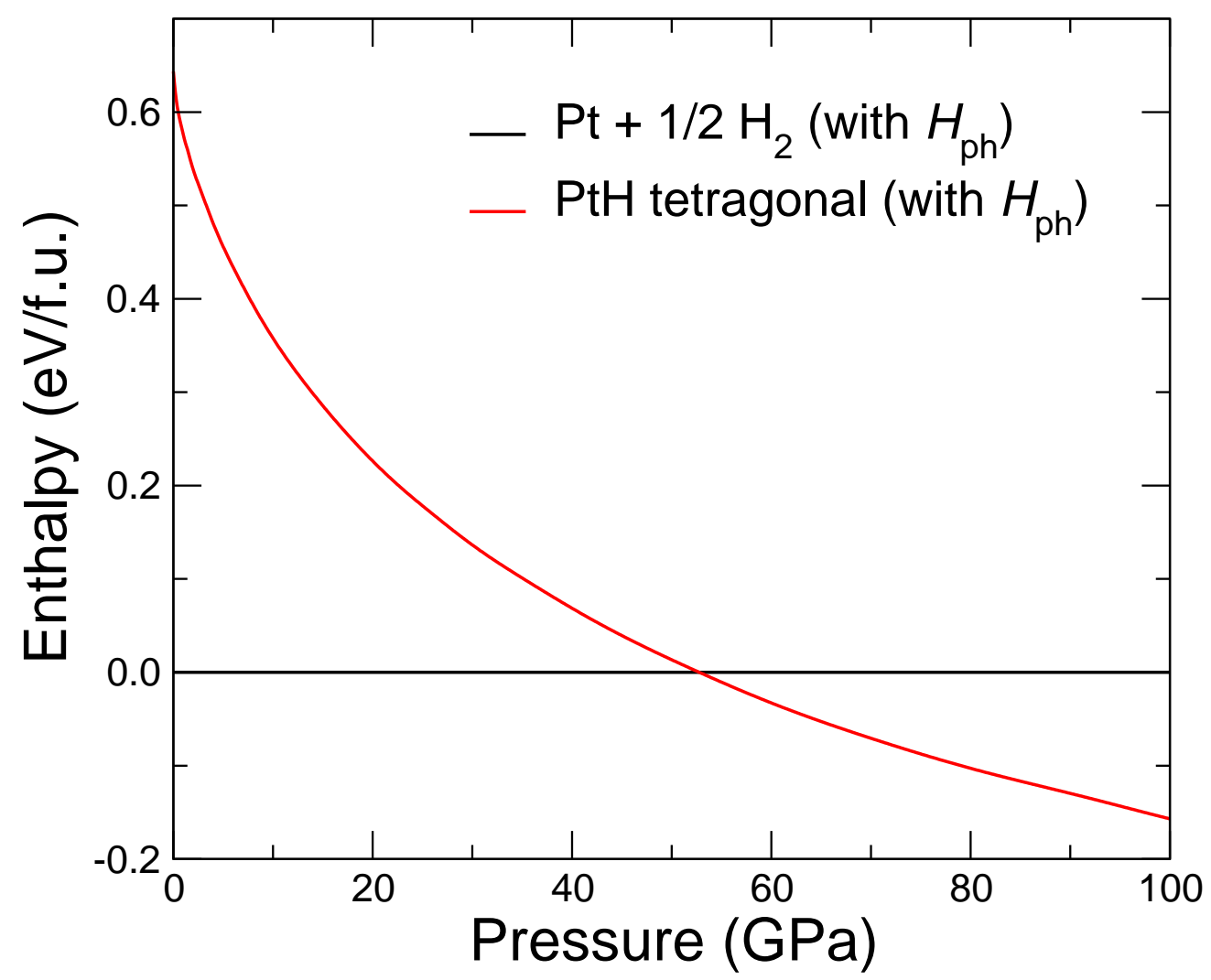

FIG. 5: Comparison of the enthalpies of $\mathrm{Pt}+\frac{1}{2} \mathrm{H}_{2}$ and the tetragonal phase of $\mathrm{PtH}$ with $H_{p h}$ included. 


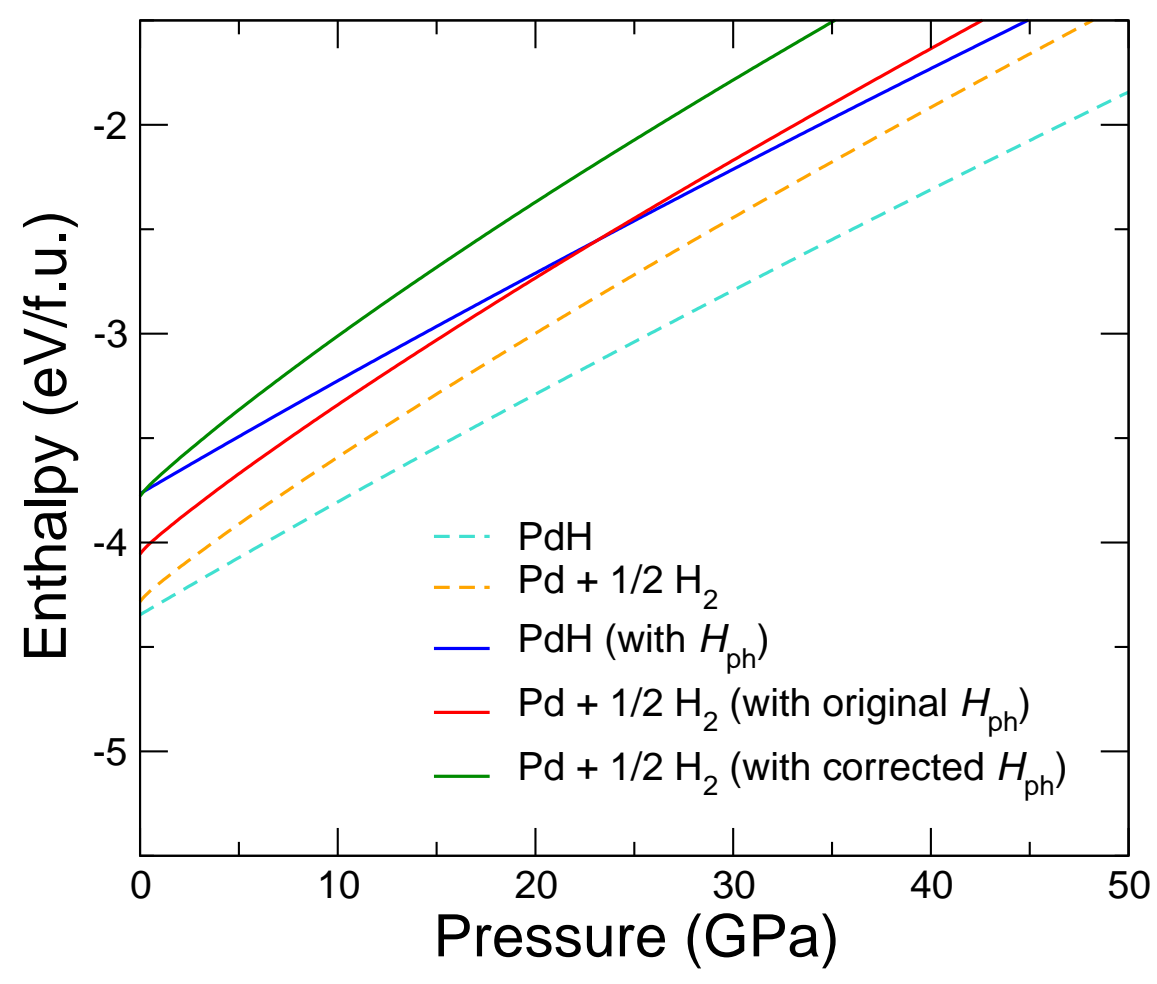

FIG. 6: Comparison of the enthalpies of $\mathrm{Pd}+\frac{1}{2} \mathrm{H}_{2}$ and $\mathrm{PdH}$ without $H_{p h}$, with $H_{p h}$, and with the corrected $H_{p h}$ contribution. 


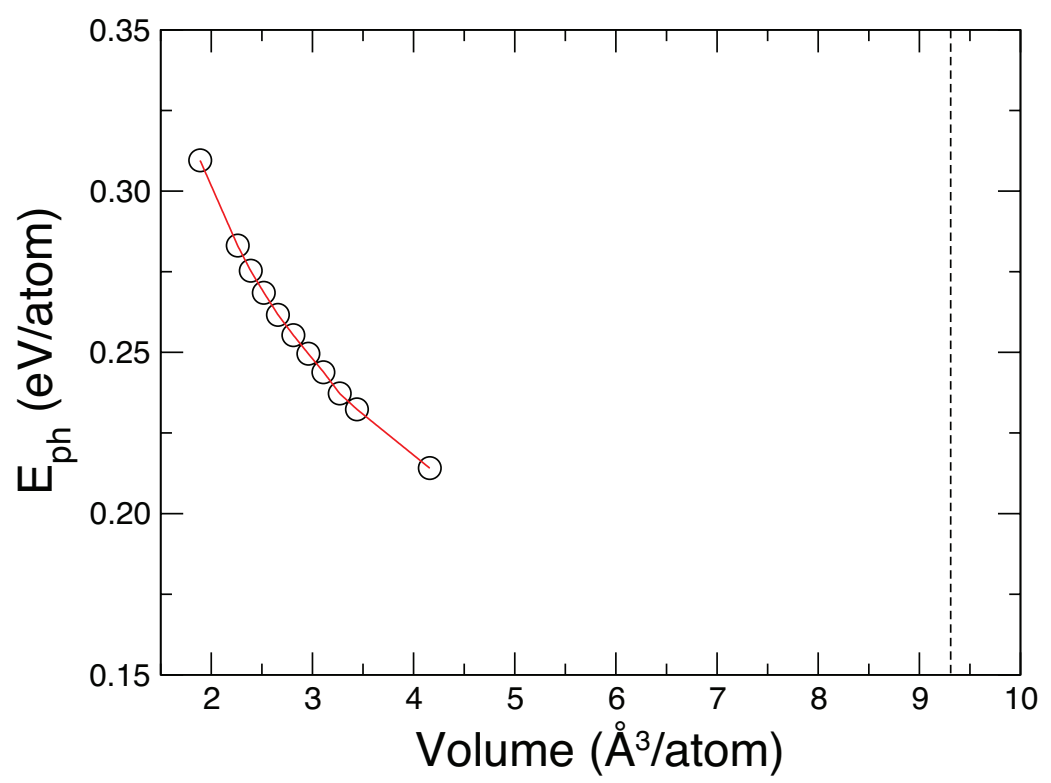

FIG. 7: $E_{p h}$ for solid $\mathrm{H}_{2}$ is plotted as a function of volume. The calculated data are shown as black circles. The connecting red lines are merely a guide to the eye. The vertical dashed line indicates the equilibrium volume of hydrogen at zero pressure. 


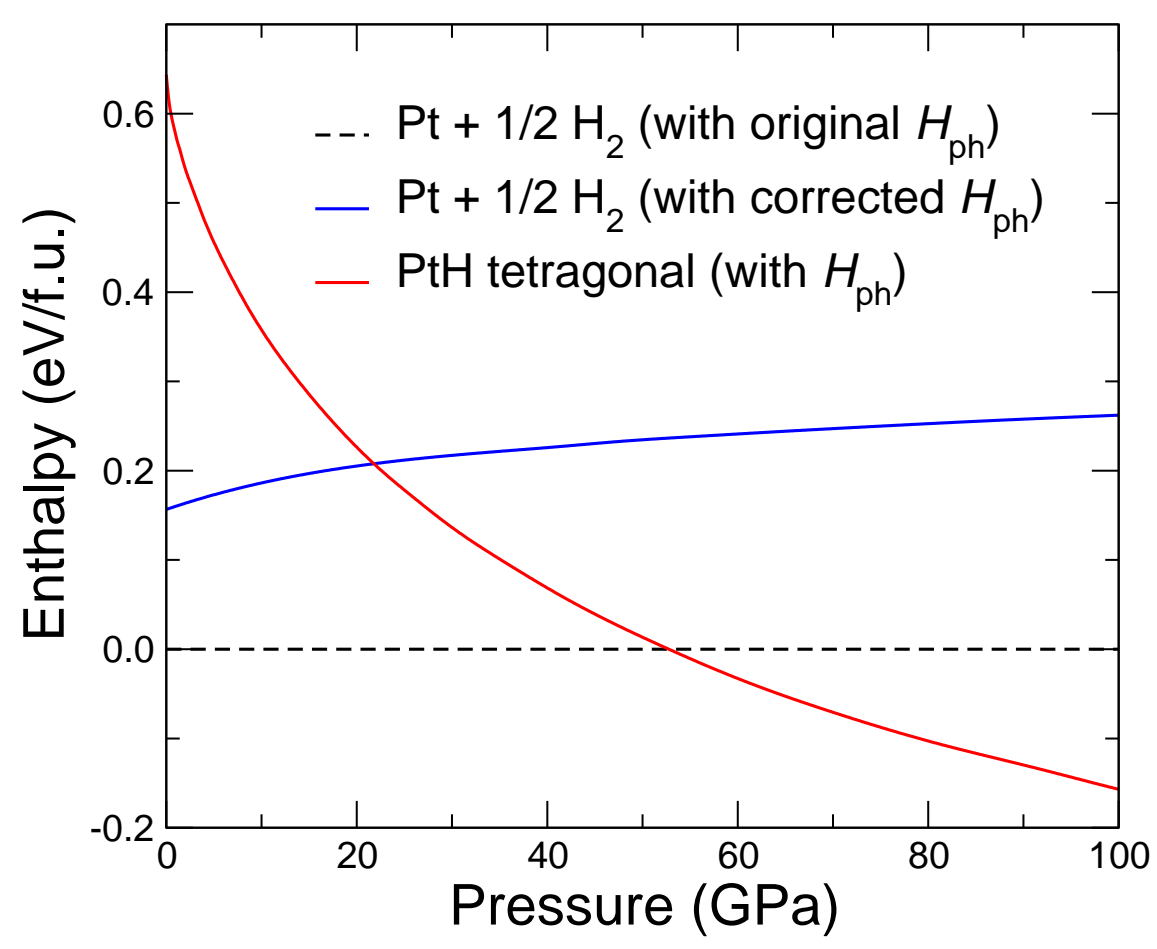

FIG. 8: Comparison of the enthalpies of $\mathrm{Pt}+\frac{1}{2} \mathrm{H}_{2}$ and tetragonal-PtH, including the correction discussed in the text. 
[1] P. Hohenberg and W. Kohn Phys. Rev. 136, B864-B871 (1964).

[2] W. Kohn and L. J. Sham Phys. Rev. 140, A1133-A1138 (1965).

[3] J. P. Perdew, J. A. Chevary, S. H. Vosko, K. A. Jackson, M. R. Pederson, D. J. Singh, and C. Fiolhais Phys. Rev. B 46, 6671-6687 (1992).

[4] J. P. Perdew, K. Burke, and M. Ernzerhof Phys. Rev. Lett. 77 3865-3868 (1996).

[5] S. J. Clark, S. J. et al. Z. Kristallogr. 220, 567-570 (2005).

[6] D. Vanderbilt Phys. Rev. B 41, 7892-7895 (1990).

[7] G. Kresse and J. Furthmüller Phys. Rev. B 54, 11169-11186 (1996).

[8] G. Kresse and J. Furthmüller Comput. Mater. Sci. 6, 15-50 (1996).

[9] P. E. Blöchl Phys. Rev. B 50 17953-17979 (1994).

[10] H. J. Monkhorst, and J. D. Pack Phys. Rev. B 13, 5188-5192 (1976).

[11] S. Baroni, S. de Gironcol, A. Dal Corso, and P. Giannozzi Rev. Mod. Phys. 73, 515-562 (2001).

[12] P. B. Allen and R. C. Dynes Phys. Rev. B 12, 905-922 (1975).

[13] W. L. McMillan Phys. Rev. 167, 331-344 (1968).

[14] B. Stritzker and W. Buckel Z. Physik 257, 1-8 (1972).

[15] D. M. Ceperley and B. J. Alder Phys. Rev. B 36, 2092-2106 (1987). 